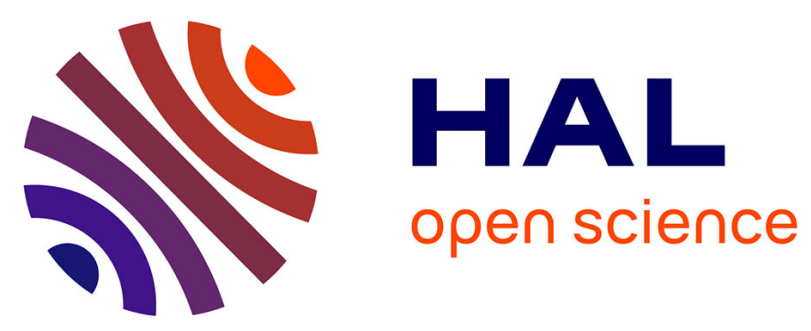

\title{
Analyzing Oxidative Stress in Murine Intestinal Organoids using Reactive Oxygen Species-Sensitive Fluorogenic Probe
}

\author{
Aline Stedman, Antonin Levy, Philippe Sansonetti, Giulia Nigro
}

\section{To cite this version:}

Aline Stedman, Antonin Levy, Philippe Sansonetti, Giulia Nigro. Analyzing Oxidative Stress in Murine Intestinal Organoids using Reactive Oxygen Species-Sensitive Fluorogenic Probe. Journal of visualized experiments : JoVE, 2021, 175, 10.3791/62880 . pasteur-03584354

\section{HAL Id: pasteur-03584354}

\section{https://hal-pasteur.archives-ouvertes.fr/pasteur-03584354}

Submitted on 22 Feb 2022

HAL is a multi-disciplinary open access archive for the deposit and dissemination of scientific research documents, whether they are published or not. The documents may come from teaching and research institutions in France or abroad, or from public or private research centers.
L'archive ouverte pluridisciplinaire HAL, est destinée au dépôt et à la diffusion de documents scientifiques de niveau recherche, publiés ou non, émanant des établissements d'enseignement et de recherche français ou étrangers, des laboratoires publics ou privés. 
TITLE:

2 Analyzing Oxidative Stress in Murine Intestinal Organoids Using Reactive Oxygen Species-

3 Sensitive Fluorogenic Probe

${ }^{1}$ Molecular Microbial Pathogenesis Unit, Institut Pasteur, INSERM U1202, 75015 Paris, France

${ }^{2}$ Chaire de Microbiologie et Maladies Infectieuses, Collège de France, 75231 Paris, France

Present address:

aSorbonne Université, CNRS UMR7622, INSERM U1156, Institut de Biologie Paris Seine (IBPS) Developmental Biology Unit, 75005 Paris, France.

bMolecular Radiotherapy, INSERM U1030, Gustave Roussy, Université Paris-Saclay, F-94805 Villejuif, France

'The Center for Microbes, Development and Health, Institut Pasteur Shanghai and Chinese Academy of Sciences, Shanghai, China

${ }^{\mathrm{d} M i c r o e n v i r o n m e n t}$ and Immunity Unit, Institut Pasteur, INSERM U1224, 75015 Paris, France

Email addresses of the authors:

Aline Stedman

(aline.stedman@sorbonne-universite.fr)

Antonin Levy (antonin.levy@gustaveroussy.fr)

Philippe J. Sansonetti

(philippe.sansonetti@ips.ac.cn)

(giulia.nigro@pasteur.fr)

*Email address of the corresponding author:

(giulia.nigro@pasteur.fr)

\section{KEYWORDS:}

Murine intestinal organoids, ROS detection, Flow cytometry analysis, Live imaging detection, intestinal stem cells, ROS-sensitive dye, oxidative stress, fluorogenic probes

\section{SUMMARY:}

The present protocol describes a method to detect reactive oxygen species (ROS) in the intestinal murine organoids using qualitative imaging and quantitative cytometry assays. This work can be potentially extended to other fluorescent probes to test the effect of selected compounds on ROS.

\section{ABSTRACT:}

Reactive oxygen species (ROS) play essential roles in intestinal homeostasis. ROS are natural byproducts of cell metabolism. They are produced in response to infection or injury at the mucosal level as they are involved in antimicrobial responses and wound healing. They are also critical secondary messengers, regulating several pathways, including cell growth and differentiation. On 
and favor intestinal diseases like chronic inflammation or cancer. This work provides a straightforward method to detect ROS in the intestinal murine organoids by live imaging and flow cytometry, using a commercially available fluorogenic probe. Here the protocol describes assaying the effect of compounds that modulate the redox balance in intestinal organoids and detect ROS levels in specific intestinal cell types, exemplified here by the analysis of the intestinal stem cells genetically labeled with GFP. This protocol may be used with other fluorescent probes.

\section{INTRODUCTION:}

Reactive oxygen species (ROS) are natural by-products of cellular metabolism. They can also be actively produced by specialized enzymatic complexes such as the membrane-bound NADPHOxidases (NOX) and Dual Oxidases (DUOX), which generate superoxide anion and hydrogen peroxide $^{1}$. By expressing antioxidant enzymes and ROS scavengers, cells can finely tune their redox balance, thereby protecting tissue homeostasis ${ }^{2}$. Although ROS can be highly toxic to the cells and damage DNA, proteins, and lipids, they are crucial signaling molecules ${ }^{2}$. In the intestinal epithelium, moderate ROS levels are required for stem and progenitor cell proliferation ${ }^{3}$; high ROS levels lead to their apoptosis ${ }^{4}$. Chronic oxidative stress is linked to many gastrointestinal diseases, such as inflammatory bowel diseases or cancer. As an example, in a mouse model of Wnt-driven intestinal cancer, elevated ROS production through activation of NADPH-oxidases was found to be required for cancer cells hyperproliferation ${ }^{5,6}$. Defining how intestinal cells and, in particular, stem cells manage oxidative stress and how the cellular environment can impact this capacity is essential to understand the etiology of this disease better?

In a tissue, different cell types present a basal oxidative state that may vary according to their function and metabolism and the expression of varying levels of oxidant and antioxidant molecules $^{4,7}$. Monitoring ROS in vivo is very challenging. Cell permeable dyes that emit fluorescence according to their redox state have been developed to visualize and measure cellular ROS in living cells and animals. However, their efficacy depends on their diffusion inside living tissues and their rapid readout, making them difficult to use in animal models ${ }^{8}$.

73

In the past, the study of the effect of compounds on ROS generation was done using cell lines, but this may not reflect the in vivo situation. The intestinal organoid model, developed by the group of Clevers $^{9}$, enables the growth of intestinal primary cells ex vivo. Culture of intestinal crypts in matrices, in the presence of defined growth factors, leads to three-dimensional structures, called organoids (mini-gut), which reproduce the crypt-villus organization, with cells from the different epithelial lineages lining an internal lumen, and the intestinal stem cells residing in small crypts-like protrusions.

Here, taking advantage of this model, a simple method is described to study oxidative stress in primary intestinal cells at the single-cell resolution by adding a commercially available ROSsensitive dye into the organoid culture medium.

Plate readers are often used to detect ROS production in a total population. This protocol uses 88 flow cytometry or imaging assay to detect ROS in a particular cell type with genetically modified cells or specific antibody staining. This work involves mouse intestinal organoid culture and ROS 
visualization by confocal imaging and quantification by flow cytometry. Using Lgr5-GFP micederived small intestinal organoids, it has been shown that it is possible to specifically analyze the level of oxidative stress in intestinal stem cells upon different treatments. This protocol can be adapted to test the influence of exogenous molecules, such as microbiota-derived muramyldipeptide $(M D P)^{10}$, on the ROS balance, after stimulating organoids with the selected compounds.

\section{PROTOCOL:}

All animal experiments were carried out after approval by the Institut Pasteur Use Committee and by the French Ministry of Agriculture no. 2016-0022. All the steps are performed inside a tissue culture hood.

\section{Preparation of reagents and materials for culturing intestinal organoids}

1.1. To prepare growth culture medium, mix advanced DMEM/F-12 supplemented with $1 x$ glutamine, $1 x$ penicillin/streptomycin (P/S) solution, $10 \mathrm{mM}$ of HEPES, $50 \mathrm{ng} / \mathrm{mL}$ of murine EGF, $20 \mu \mathrm{g} / \mathrm{mL}$ of murine Noggin $500 \mathrm{ng} / \mathrm{mL}$ of mouse R-Spondin1 (see Table of Materials). Leave the medium at room temperature (RT) during the crypt's extraction.

NOTE: Freeze the unused medium in aliquots at $-20^{\circ} \mathrm{C}$. Avoid freeze and thaw.

1.2. Fill a $50 \mathrm{~mL}$ tube with $40 \mathrm{~mL}$ of Advanced DMEM/F-12 and keep it on ice.

NOTE: Keep the unused medium at $4{ }^{\circ} \mathrm{C}$. It will be used for organoids passaging.

1.3. Pre-warm the cell culture plates ( $\mu$-Slide 8 well chambers and/or 96 -well round bottom) in the incubator at $37^{\circ} \mathrm{C}$.

1.4. Thaw basement membrane matrix (BMM) (see Table of Materials) aliquots on ice (before starting the protocol or at least $1 \mathrm{~h}$ before plating crypts).

NOTE: The BMM will quickly solidify if not kept cold.

1.5. Prepare washing/flushing solution adding $1 \%$ penicillin-streptomycin solution to DPBS (DPBS-P/S).

1.6. Fill a $100 \mathrm{~mm}$ petri-dish with $10 \mathrm{~mL}$ of cold DPBS-P/S. Fill six $15 \mathrm{~mL}$ tubes with $10 \mathrm{~mL}$ of DPBS and label them from F1 to F6.

1.7. Prepare $30 \mathrm{~mL}$ of $10 \mathrm{mM}$ EDTA solution by dilution from $0.5 \mathrm{M}$ EDTA in DPBS. Fill two 15 $\mathrm{mL}$ tubes with $10 \mathrm{~mL}$ of $10 \mathrm{mM}$ EDTA, and label them E1 and E2.

1.8. Keep all solutions pre-cooled at $4^{\circ} \mathrm{C}$ and keep them on the ice during the procedure. 


\section{Intestinal organoids culture}

2.1. Sacrifice a 8-10 weeks-old Lgr5-EGFP-IRES-creERT2 (Lgr5-GFP) mouse according to the national rules and regulations.

2.2. Collect $5-8 \mathrm{~cm}$ of the jejunum encompassing the region between the duodenum $(5 \mathrm{~cm}$ from the stomach) and the ileum (10 cm from the cecum) and keep in cold DPBS-P/S on ice.

2.3. Clean the intestinal content by flushing with $5-10 \mathrm{~mL}$ of cold DPBS-P/S.

NOTE: Home-made flushing syringes can be obtained by plugging a $200 \mu \mathrm{L}$ tip onto a $10 \mathrm{~mL}$ syringe nozzle.

2.4. Open the intestine longitudinally using ball tip scissors (see Table of Materials) (to prevent damaging the tissue).

2.5. Using forceps, transfer the tissue into a petri dish containing cold DPBS-P/S at room temperature and shake it to rinse.

2.6. With a plastic Pasteur pipette, grab the intestine by aspiration and transfer it into a $15 \mathrm{~mL}$ tube labeled E1 containing $10 \mathrm{~mL}$ cold $10 \mathrm{mM}$ EDTA.

2.7. Invert the tube 3 times and incubate on ice for $10 \mathrm{~min}$.

2.8. Using a plastic Pasteur pipette, transfer the tissue in tube $\mathbf{F 1}$ containing $10 \mathrm{~mL}$ DPBS. Vortex for 2 min (on normal vortex, holding the tube by hand and ensuring that the intestine swirls nicely).

2.9. Put $10 \mu \mathrm{L}$ of the fraction in a petri dish and assess the quality of the fraction under a microscope.

NOTE: All vortex steps are performed at maximum speed, and the quality of each fraction should be assessed under the microscope (Figure 1).

2.10. With a plastic Pasteur pipette, grab the intestine by aspiration and transfer it in tube $\mathbf{F} \mathbf{2}$ containing $10 \mathrm{~mL}$ DPBS and vortex for $2 \mathrm{~min}$.

2.11. Repeat step 2.10, transferring the tissue in tube $\mathbf{F 3}$ containing $10 \mathrm{~mL}$ DPBS and vortex for $2 \mathrm{~min}$.

2.12. Repeat EDTA incubation as in step 2.6, transferring the tissue in tube E2 containing 10 mM EDTA.

2.13. Invert the tube 3 times and incubate on ice for $5 \mathrm{~min}$. 
2.14. Repeat step 2.10, transferring the tissue in tube $\mathbf{F 4}$ containing $10 \mathrm{~mL}$ DPBS and vortex for $3 \mathrm{~min}$.

2.15. Repeat step 2.14, transferring the tissue in tube $\mathbf{F 5}$ containing $10 \mathrm{~mL}$ DPBS and vortex for $3 \mathrm{~min}$.

2.16. Repeat step 2.15, transferring the tissue in tube $\mathbf{F 6}$ containing $10 \mathrm{~mL}$ DPBS and vortex for $3 \mathrm{~min}$.

2.17. Combine the best fractions filtering by gravity through a $70 \mu \mathrm{m}$ cell strainer into a $50 \mathrm{~mL}$ tube (on ice) to eliminate villi and significant debris.

NOTE: Usually, F5 and F6 are the fractions containing numerous crypts and less debris.

\subsection{Spin the crypts at $150 \times g$ at $4^{\circ} \mathrm{C}$ for $3 \mathrm{~min}$.}

2.19. Empty the tube, disrupt the pellet mechanically, and add $5 \mathrm{~mL}$ of cold DMEM/F12.

2.20. Put $10 \mu \mathrm{L}$ of the suspension in a petri dish and count the number of crypts present in the aliquot manually under a microscope.

NOTE: Do not count single cells or small debris.

2.21. Calculate the volume $(\mathrm{V})$ of crypts suspension required in $\mu \mathrm{L}$, considering that 300 crypts are plated per well, $\mathrm{W}$ is the number of wells, and $\mathrm{N}$ is the number of crypts counted out of 10 $\mu \mathrm{L}$ of the suspension.

NOTE: $V=300 \times W \times 10 / N$. Then transfer the solution to a new $15 \mathrm{~mL}$ tube. If a small volume is used in the planned experiment, a $1.5 \mathrm{~mL}$ centrifuge tube can be used.

\subsection{Spin the crypts at $200 \times g$ at $4^{\circ} \mathrm{C}$ for $3 \mathrm{~min}$.}

2.23. Carefully remove the supernatant using a pipette.

2.24. Mechanically disrupt the pellet and gently add growth culture medium to obtain a concentration of 90 crypts/ $\mu \mathrm{L}$.

2.25. Add 2 volumes of undiluted BMM to have a final concentration of $30 \mathrm{crypts} / \mu \mathrm{L}$. Carefully pipette up and down without introducing air bubbles into the mix. 
2.26. Plate $10 \mu \mathrm{L}$ of the crypts/BMM mix into each well. For Flow cytometry analysis, use roundbottom 96-well plates. Distribute $10 \mu \mathrm{L}$ at the center of each well as a dome. For imaging, use $\mu$ slide 8 well (see Table of Materials) and deposit the $10 \mu \mathrm{L}$ as a thin layer.

NOTE: Plate the organoids as a thin layer for the imaging assay to enable their in-depth imaging.

2.27. Leave the plate for $5 \mathrm{~min}$ at RT to allow the BMM to solidify. Place the plate in the

\subsection{Add $250 \mu \mathrm{L}$ of growth medium into each well, taking care not to detach the BMM.}

2.29. Place the plates in the incubator at $37{ }^{\circ} \mathrm{C}$ and $5 \% \mathrm{CO}_{2}$.

2.30. Perform the ROS analysis between days 4 and 6 of culture. Otherwise, change the medium and split the organoids after the appearance of several and long budding structures and when dead cells accumulate into the organoids lumens.

\section{Organoids passaging}

3.1. Start passaging small intestinal organoids from the $6^{\text {th }}$ day of culture, when significant budding structures have formed, and the organoids lumens have become dark.

NOTE: The organoid's lumen becomes dark due to the accumulation of dead cells, debris, and mucus. Avoid letting the organoids overgrow before splitting. The splitting ratio depends on the organoids' growth. Passaging the organoids with a ratio of $1: 2$ at day 6 and $1: 3$ at day 10 is recommended.

\subsection{Fill a $15 \mathrm{~mL}$ tube with $4 \mathrm{~mL}$ of cold Advanced DMEM/F-12 and keep it on ice.}

NOTE: Here, volumes for a 96-well culture plate are provided. If a different format is used, adjust the volume accordingly.

3.3. Carefully aspirate the medium with a pipette or a vacuum pump from the wells without touching the BMM domes, and discard it.

3.4. Add $100 \mu \mathrm{L}$ of cold Advanced DMEM/F-12 per well. Pipette up and down to break the $\mathrm{BMM}$ and transfer the content of the well into the $15 \mathrm{~mL}$ tube.

3.5. Wash the well with $200 \mu \mathrm{L}$ cold Advanced DMEM/F-12 and collect it in the same tube.

NOTE: If passaging multiple wells from the same experimental condition, the contents of the wells can be pooled in the same $15 \mathrm{~mL}$ collecting tube.

3.6. Spin the $15 \mathrm{~mL}$ collecting tube at $100 \times g$ for $5 \mathrm{~min}$ at $4^{\circ} \mathrm{C}$. 
3.7. Discard the supernatant and add $1 \mathrm{~mL}$ of cold Advanced DMEM/F-12 to the pellet. Using a P1000 tip, take up a P10 tip (without filter) and pipette up and down at least 20 times.

3.8. Add $4 \mathrm{~mL}$ of cold Advanced DMEM/F-12 to the tube. Spin at $300 \times g$ for $5 \mathrm{~min}$ at $4^{\circ} \mathrm{C}$.

3.9. Aspirate the supernatant with a pipette or a vacuum pump without disturbing the pellet. Then, disrupt the pellet mechanically.

3.10. Add BMM diluted in growth culture medium (2:1 ratio). Carefully pipette up and down without introducing air bubbles into the mix.

3.11. Plate $10 \mu \mathrm{L}$ of the crypts/BMM mix into each well.

3.12. Keep the plate for $5 \mathrm{~min}$ at RT to allow the BMM to solidify. Place the plate in the incubator at $37{ }^{\circ} \mathrm{C}$ and $5 \% \mathrm{CO}_{2}$ for $15 \mathrm{~min}$.

3.13. Add $250 \mu \mathrm{L}$ of growth medium into each well.

NOTE: Be careful not to detach the BMM.

3.14. Place the plates in the incubator at $37^{\circ} \mathrm{C}$ and $5 \% \mathrm{CO}_{2}$.

\section{Preparation of reagents and materials to assess oxidative stress in intestinal organoids}

4.1. Prepare a $250 \mathrm{mM}$ stock solution of inhibitor $\mathrm{N}$-acetylcysteine (NAC) (see Table of Materials), resuspend $10 \mathrm{mg}$ with $245 \mu \mathrm{L}$ of DPBS. Use at $1 \mathrm{mM}$ final concentration.

4.2. Prepare a $50 \mathrm{mM}$ stock solution of inducer Tert-butyl hydroperoxide (tBHP), 70\% in water, dilute $3.22 \mu \mathrm{L}$ with $496.8 \mu \mathrm{L}$ of DPBS. Use at $200 \mu \mathrm{M}$ final concentration.

4.3. For the Flow cytometry study, prepare a $250 \mu \mathrm{M}$ working solution of a fluorogenic probe (see Table of Materials) by diluting the stock solution $1 / 10$ in DMSO. Use at $1 \mu \mathrm{M}$ final concentration.

NOTE: As indicated in the manufacturer's instructions, the fluorogenic probe is sensitive to light and oxygen. Stocks and aliquots should not be open and close too many times.

4.4. For the imaging study, prepare a $1.25 \mathrm{mM}$ working solution of the fluorogenic probe by diluting the stock solution $1 / 2$ in DMSO. Use at $5 \mu \mathrm{M}$ final concentration.

4.5. Prepare a final solution of $0.1 \mu \mathrm{g} / \mathrm{mL}$ DAPI in DPBS, to be used for dead cell discrimination in the Flow cytometry assay. 
4.6. Dilute Hoechst 33342 to $1.25 \mathrm{mg} / \mathrm{mL}$ in DPBS. Use at $5 \mu \mathrm{g} / \mathrm{mL}$ final concentration to be used for nuclear staining in the imaging assay.

\subsection{Warm DMEM without phenol red at $37^{\circ} \mathrm{C}$.}

NOTE: These steps describe using negative and positive controls that must be included in any assays, using the conditions indicated in Figure 2A. The assay can be used to test anti- or prooxidant compounds. The steps are the same, and the only difference is when the compounds are added before using the fluorogenic dye.

\section{Visualization of oxidative stress in 3D organoids by confocal microscopy}

5.1. Take the organoids plated in the $\mu$-Slide 8 well chambers and add $1 \mu \mathrm{L}$ NAC stock solution in the corresponding wells to obtain a final concentration of $1 \mathrm{mM}$.

5.2. Incubate for $1 \mathrm{~h}$ at $37^{\circ} \mathrm{C}$ and $5 \% \mathrm{CO}_{2}$.

5.3. Add $1 \mu \mathrm{L}$ tBHP stock solution in the corresponding wells to obtain a final concentration of $200 \mu \mathrm{M}$.

5.4. Incubate for $30 \mathrm{~min}$ at $37^{\circ} \mathrm{C}$ and $5 \% \mathrm{CO}_{2}$.

5.5. Add $1 \mu \mathrm{L}$ per well of the $1.25 \mathrm{mM}$ dilution of the fluorogenic probe to obtain a final concentration of $5 \mu \mathrm{M}$.

5.6. Add $1 \mu \mathrm{L}$ per well of the $1.25 \mathrm{mg} / \mathrm{mL}$ dilution of Hoescht to obtain a final concentration of $5 \mu \mathrm{g} / \mathrm{mL}$.

5.7. Incubate for $30 \mathrm{~min}$ at $37^{\circ} \mathrm{C}$ and $5 \% \mathrm{CO}_{2}$.

5.8. Remove the medium without disturbing the BMM. Gently, add $250 \mu \mathrm{L}$ of warm DMEM without phenol red.

NOTE: If a long-term acquisition is planned, add growth factors compounds to DMEM without phenol red.

5.9. Image the organoids using a confocal microscope equipped with a thermic chamber and gas supply that detects the fluorogenic probe (ROS).

NOTE: The excitation/emission (ex/em) for the fluorogenic probe is $644 / 665$, ex/em for Hoechst (nuclei) is 361/486, and ex/em for GFP (intestinal stem cell from the Lgr5-GFP mice) is 488/510. A $63 x$ oil immersion objective is used to detect signals in stem cells. Do not change laser settings between samples. A 20x objective might be used to allow an overview of ROS production. 
5.10. Use the positive control to set up laser intensity and time exposure for the ROS signal and check that this signal is lower in the negative control.

\subsection{Using eyepiece screen the slide to identify the organoids expressing GFP and adjust laser}

NOTE: This step is manually performed. The eyepiece screens the slide to identify the GFP expressing organoids.

5.12. Define positions to obtain a stitched image of the whole organoid. Setup a z-stack of 25 $\mu \mathrm{m}$ (step size $5 \mu \mathrm{m}$ ) to get a section of the organoids showing one layer of cells.

NOTE: Refer to the microscope user manual to optimize the setup. Using living cells, the acquisition should be done within $1 \mathrm{~h}$ after the end of the incubation.

\subsection{Open the images in an open-source image processing software (see Table of Materials).}

5.14. Go through the z-stack and choose the section in which the middle of the organoids is well represented and create a new image with the selected area.

\subsection{Quantify the images as per steps 5.15.1-5.15.5.}

5.15.1. Select the freehand line tool.

5.15.2. Draw a line following the nuclei.

NOTE: Select only regions presenting GFP-positive cells if only stem cells are analyzed.

5.15.3. Increase the line width to cover the cell layer with the line without including the luminal debris.

5.15.4. Select the channel for the ROS signal and measure the fluorescence intensity in the selected region and annotate the values.

5.15.5. Draw a line where there is no signal and measure the fluorescent intensity of the background that will be subtracted to the previous value to get the final intensity.

\section{Quantification of the oxidative stress on the dissociated organoids using flow cytometer}

6.1. Add $1 \mu \mathrm{L}$ NAC stock solution in the wells for negative controls to obtain a final concentration of $1 \mathrm{mM}$.

NOTE: Use the organoids plated in the 96-well round-bottom plates. 
6.2. Incubate for $1 \mathrm{~h}$ at $37^{\circ} \mathrm{C}$ and $5 \% \mathrm{CO}_{2}$.

6.3. Add $1 \mu \mathrm{L}$ tBHP stock solution in the corresponding wells to obtain a final concentration of $200 \mu \mathrm{M}$.

6.4. Incubate for $30 \mathrm{~min}$ at $37^{\circ} \mathrm{C}$ and $5 \% \mathrm{CO}_{2}$.

6.5. With a multichannel pipette, remove the medium without disturbing the attached BMM and transfer it to another 96 -well round bottom plate. Keep this plate aside.

6.6. Add $100 \mu \mathrm{L}$ of trypsin, and with a multichannel pipette, pipette up and down at least five times to destroy the BMM.
6.7. Incubate for not more than $5 \mathrm{~min}$ at $37^{\circ} \mathrm{C}$ and $5 \% \mathrm{CO}_{2}$.

6.8. With a multichannel pipette, pipette up and down at least five times to dissociate the organoids.

\subsection{Spin at $300 \times g$ for 5 min at RT.}

6.10. Discard the supernatant by inverting the plate. Add back the medium collected in step 6.3 to the corresponding wells and resuspend the cells by pipetting up and down 5 times.

6.11. Add the fluorogenic probe at the final concentration of $1 \mu \mathrm{M}$. Add $1 \mu \mathrm{L}$ per well from the $250 \mu \mathrm{M}$ dilution and incubate for $30 \mathrm{~min}$ at $37^{\circ} \mathrm{C}$ and $5 \% \mathrm{CO}_{2}$.

NOTE: Do not add the fluorogenic probe to the wells needed for the instrument's settings (Figure 2B).

\subsection{Spin at $300 \times g$ for 5 min at RT.}

6.13. Resuspend the cells with $250 \mu \mathrm{L}$ of $0.1 \mu \mathrm{g} / \mathrm{mL}$ DAPI solution. Transfer the samples in the proper Flow cytometry tubes, keep the tubes on ice, and proceed with the analysis.

NOTE: Add PBS instead of DAPI to the wells needed for the instrument's settings (Figure 2B).

6.14. Optimize the forward and side scatter voltage settings on unstained control and laser voltages for each fluorophore using mono-stained samples.

6.15. Using an appropriate gating strategy (Figure 4A), collect a minimum of 20,000 events.

NOTE: 50,000 events are preferred. Detailed acquisition settings vary according to the instrument used. 
441 As a proof of concept of the described protocol, the crypts obtained from the Lgr5-eGFP-IRESCreERT2 mouse line were used in which intestinal stem cells display mosaic GFP expression, which was established by Barker et al., to characterize intestinal stem cells ${ }^{10}$ initially and allow to map these cells based on their GFP expression. A model is thereby provided to compare ROS levels in a specific cell type population upon different treatments. A ROS inhibitor (NAC) was used, and an inducer (tBHP), known to act on cellular ROS to visualize changes in their levels.

Figures $1 \mathrm{~A}$ and $1 \mathrm{~B}$ show representative images of fractions F1 and F4 obtained during the crypts extraction procedure for the intestinal organoid culture. Each fraction must be checked under a microscope or binocular during the extraction procedure to follow crypts detachment and define those fractions enriched in crypts, rather than villi, single cells, or debris. The chosen fractions are then pooled together and passed through a $70 \mu \mathrm{m}$ cell strainer to remove all the remaining fragments of villi and obtain a preparation with only crypts (Figure 1C). The crypts start to close within a few hours of embedding in BMM, and at D1, round organoids were observed (Figure 1D). After 3-5 days, the organoids will appear with budding structures representing the "newly formed crypts." The organoids are ready for ROS analysis (Figures $1 \mathbf{E}$ and 1F).

In the protocol of imaging oxidative stress by confocal microscopy, the slide containing the organoids, incubated with the probe, was imaged with a confocal fluorescence microscope equipped with lasers and filters to detect the Hoechst (ex/em: 361/486), the GFP (ex/em: 488/510) and the fluorogenic probe (ex/em): 644/665) signals. A confocal microscope equipped with 20x air and 63x oil immersion objective allowed the visualization of ROS. In Lgr5-GFP mice, the GFP-positive cells are Lgr5-expressing intestinal stem cells. Supplementary Figure 1 shows representative images obtained with the 20x objective providing an overview of the ROS in several organoids. Figure 3 shows representative images, obtained with the $63 x$ oil objective, of intestinal organoids expressing GFP, non-treated (NT), or pre-incubated or not with the ROS inhibitor NAC, and stimulated or not for 30 min with the ROS inducer tBHP.

In the presence of the inhibitor, the only signal from the dead cells contained in the lumen of the organoid is visible. In the non-treated organoid, the basal ROS levels are shown, proving that stem cells produce higher ROS than differentiated cells (according to the microscope settings, the ROS signal might also be visualized in non-stem cells). GFP-positive cells present a more significant cytoplasmic signal with the inducer in the presence of the fluorogenic probe, demonstrating that ROS levels increase particularly in stem cells after treatment.

Figure 4 shows representative results obtained when analyzing ROS production in intestinal organoids stimulated or not with ROS inhibitor or inducer, using a Flow cytometer equipped with $405 \mathrm{~nm}, 488 \mathrm{~nm}$, and $630 \mathrm{~nm}$ lasers. The gating strategy presented in Figure 4A makes it possible to evaluate ROS production at the level of the whole organoids cell population, defining intact and living cells based on physical parameters and DAPI exclusion (SSC-A vs. FSC-A and DAPI vs. FSC-A) and FSC-H vs. FSC-A) or only in the intestinal stem cells, further gated on cells with GFP high signal. Figure 4B shows the ROS levels in the total population upon collection of 50,000 events. Basal ROS levels in the non-treated (NT) cells decrease after stimulation with the inhibitor 
(NAC), and on the contrary, increase after challenge with the inducer (tBHP). Cells pre-treated with the inhibitor and then stimulated with the inducer present a lower level than those stimulated with the inducer alone. The results were then analyzed using appropriate software, obtaining the median fluorescent intensity (MFI). The obtained values are presented as a ratio over the non-treated cells, as shown in the graph presented on the right of Figure 4B. Figure 4C shows the same parameters described in Figure 4B in the stem cells, gated as GFP positive cells, showing a 3.5-fold decrease in ROS level upon NAC treatment and 4-fold increase upon tBHP treatment over non-stimulated cells. This result demonstrates that following this protocol, it is possible to quantify differences in ROS levels at the level of the whole cell population or in GFP positive stem cells upon their treatment of the organoids with specific compounds.

\section{FIGURE LEGENDS:}

Figure 1: Representative images of crypts and organoids. (A) Example of fraction F1 obtained after the first incubation with EDTA, enriched in villi (square), with some debris (star) and crypts (circle). (B) Example of fraction F4 enriched in crypts. (C) Suspension presenting only isolated crypts obtained after the filtration with a $70 \mu \mathrm{m}$ cell strainer (scale bar, $200 \mu \mathrm{m}$ ). (D, E, and F). Typical organoids were obtained after 1, 3, and 5 days respectively, after embedding the crypts in BMM (scale bar, $100 \mu \mathrm{m})$.

Figure 2: Outline of the experimental plan. (A) Conditions used in this protocol included in each experiment: non-treated wells (NT), inducer-treated wells (tert-Butyl hydroperoxide - tBHP), inhibitor-treated wells ( $\mathrm{N}$-acetyl cysteine - NAC), and inhibitor- and inducer-treated wells (NACtBHP). (B) Plate format for the flow cytometry assay. Each condition is plated in triplicate (line A). Lines $B, C$, and $D$ include wells for flow cytometer setting with only the fluorogenic probe, only DAPI, or non-stained (NS) samples.

Figure 3: Representative confocal images of ROS staining in organoids. Stitched images were obtained with a confocal microscope equipped with a high-speed EMCCD Camera, 63x/1.4 oil objective, and slit $35 \mu \mathrm{m}$, using the lasers 405, 488, 640, and filters 460/50, 535/50, 700/75 to acquire Hoechst, GFP, and the fluorogenic probe respectively. Confocal optical sections of organoids non-treated (NT), treated with the ROS-inhibitor (NAC), with the ROS-inducer (tBHP), or pre-treated with the ROS inhibitor and then stimulated with the ROS-inducer (NAC-tBHP). In grey, nuclei stained with Hoechst; in green, Lgr5-GFP cells; in red, the fluorogenic probe (scale bar, $50 \mu \mathrm{m})$.

Figure 4: Representative flow cytometry analysis of ROS in cells derived from organoids. (A) Schematic representation of the gating strategy used in flow cytometry analysis: gating for cell shape (exclusion of dead cells and debris accumulated in the organoids lumen), gating for living cells (cells not incorporating DAPI-laser 405), gating for single cells (doublet discrimination), and stem cells (GFP positive cells-laser 488) (FSC: forward scatter, SSC: side scatter). The ROS signal has been acquired using the 630 laser. (B) On the left, histograms were obtained with an appropriate software showing the intensity ROS signals for the total living population (after gating around 10,000 events per condition) in the different samples NT: non-treated; NAC: 
inhibitor-treated; tBHP inducer-treated; NAC-tBHP: inhibitor- and inducer-treated. On the right, a typical example of the calculated ratio for MFI values over the NT samples obtained during an experiment starting from 3 samples per condition (mean $\pm S D)(* * * P=0.0003)$. (C) Same as in $B$ for the GFP positive population (1,000 events per condition) $(* P=0.02)$.

Supplementary Figure 1: Representative confocal images of ROS staining in organoids. Stitched images were obtained with a confocal microscope equipped with a high-speed EMCCD Camera, 20x objective, and slit $35 \mu \mathrm{m}$, using the lasers 405, 488, 640, and filters 460/50, 535/50, 700/75 to acquire Hoechst, GFP, and fluorogenic probe respectively. Confocal optical sections of organoids non-treated (NT), treated with the ROS-inhibitor (NAC), with the ROS-inducer (tBHP), or pre-treated with the ROS inhibitor and then stimulated with the ROS-inducer (NAC-tBHP). In grey, nuclei stained with Hoechst; in green, Lgr5-GFP cells; in red, fluorogenic probe (scale bar, $100 \mu \mathrm{m})$.

\section{DISCUSSION:}

543 This work provides a step-by-step protocol to isolate murine jejunal crypts, culture them into 3D 544 organoids, and analyze ROS in organoids by combining a ROS-sensitive fluorogenic probe with qualitative microscopy imaging of whole organoids and quantitative ROS measurement using flow cytometry on single cells following organoid dissociation.

The first critical step in this method is the crypts extraction procedure. Indeed, the quality of crypts preparation is the key to successful organoids formation. It is therefore essential to obtain fractions with enriched crypts and few cellular debris or dying cells. The crypts may be found in different fractions to those indicated in the protocol, as dissociation may vary with the age and health status of the mouse. The number of EDTA incubations can be modified accordingly. If crypts do not seem to be detaching after fraction 4, a 3 min EDTA incubation needs to be repeated. Inversely, suppose crypts already detach after the first EDTA incubation. In that case, the second EDTA incubation may not be necessary, and the sequential vortex steps in DPBS should be done until fractions are obtained with enough crypts devoid of debris. If no dissociation occurs, make sure DPBS without $\mathrm{Ca}^{2+}$ and $\mathrm{Mg}^{2+}$ is used to prepare the collecting tubes, and replace EDTA with a new solution. Crypts are fragile structures, so they should be kept as much as possible on ice and rapidly plated after isolation.

Different plates and drop volumes may be used to cultivate organoids. For instance, crypts can also be plated in $\mathbf{2 4}$ or $\mathbf{4 8}$ well plates after adjusting the crypts concentration, the volume of the BMM drop, and the medium added in each well. Multiple drops may be plated in the same well in a 12- or 6-well plate. Generally, crypts decrease in size and round up to form small round organoids at day 1 of culture. Formation of new buds should be observed 2-3 days after the plating.

For studying changes in ROS levels in intestinal stem cells, the advantage of the Lgr5-eGFP-IRESCreERT2 mouse line was taken. A caveat of this model is the selective silencing of the knocked-in allele and the consequent mosaicism of the GFP expression, which can be absent in patches of stem cells or entire crypts. During the imaging protocol, not all the organoids will present stem 
572 cells expressing GFP; therefore, not all the organoids will be considered unless it is possible to 573 rely on the spatial position of the cells. Instead, this must be considered when analyzing the GFP

574 negative cell population in the Flow cytometry protocol. Indeed, as it is impossible to rely on the 575 spatial position, the GFP-negative population will be composed of non-stem cells and GFP576 negative stem cells.

Here a protocol is provided for the qualitative evaluation of ROS in intestinal organoids. A critical aspect for this part is linked to the working distance of the objectives that are used. The organoids are grown in BMM; they are not attached to the bottom of the well, introducing a distance from the objective focus plan. For this reason, it is critical to plate the organoids in a thin layer of BMM, to minimize this issue. Even in this optimized setting, not all the organoids will be in the correct position to be adequately imaged.

A quantitative analysis of the images might be done using an appropriate image analysis software, evaluating the mean fluorescent intensity of the images in the ROS signal channel as described in the protocol. For this purpose, it is necessary to acquire a high number of images to get a sufficient number of events to be statistically significant. As mentioned before, using the Lgr5-GFP mice, not all the organoids will express GFP, requiring a considerable number of samples to be imaged.

During the flow cytometry procedure, a critical step is the dissociation of the organoids into single cells. If the dissociation is too harsh, cells may die and release DNA. A rock-inhibitor, Y-27632, to counteract anoikis, and DNAse may be added to the dissociation buffer if they do not interfere with the studied pathway. Trypsin dilution or reduced incubation times may be used.

Finally, it is crucial to define the best time point to analyze ROS production after the different treatments (anti- or pro-oxidant) tested. In the case of drugs that rapidly induce ROS within minutes or hours, the imaging assay can be used to determine when there is the maximal induction by adding the fluorogenic probe before the tested compounds. The fluorescence intensity of the probe after organoids stimulation may vary between experiments performed on different days. Therefore, it is crucial always to calculate the ratio with the non-stimulated samples and add controls (oxidant /antioxidant) to verify the reactivity of the probe. NAC and tBHP were used as negative and positive controls as they gave the most conclusive results. Still, other reagents may be used, such as resveratrol as an antioxidant or paraquat/menadione as oxidants. Incubating cells for too long with the fluorogenic probe may be toxic and even modify the cell redox balance, so incubation times must also be tightly controlled. Cells stained with the probe may be fixed and analyzed a few hours after. In this case, for the flow cytometry analysis, the DAPI cannot discriminate between living and dead cells. Instead, a fixable dye for live/dead discrimination should be used before fixation.

614

Organoids may also be grown for several days (more than 7), but this will increase the number of living and proliferative cells and the dead cells that accumulate in the organoids lumens, generating high background, particularly in the imaging assay. If an abnormal increase in the fluorogenic probe signal is observed, ensure that the solution used to resuspend stimulating 
compounds is not pro-oxidant per se (i.e., ethanol).

One concern to consider when using this protocol is that live imaging and cell dissociation followed by flow cytometry may induce oxidative stress in cells and generate a background signal. Fixation of the organoids may be considered according to the experiment. Another limitation arises from the difficulty in the in-depth imaging of organoids grown in a 3D matrix. As mentioned in the protocol, the BMM should be distributed on the slide as a thin layer to limit this aspect.

Here, the protocol is designed using a commercially available fluorogenic dye. Its primary advantage is its compatibility with multi-color staining of organoids so that specific cell types. For instance, antibody staining for cell surface markers immediately after the fluorogenic probe incubation may be done to detect particular sub-types. However, the probe is not specific to a specific ROS species as it can detect Superoxide, Nitrite peroxide, and hydrogen peroxide ${ }^{11-13}$. For this reason, it is generally used to detect global oxidative stress. Although commercialized as a cytosolic-only probe, the selected fluorogenic probe could be found to reach mitochondria ${ }^{14}$. As its specificity can vary between different cellular contexts, we suggest using other complementary approaches to measure ROS when possible. Alternative dyes such as probes specific to detect mitochondria-generated superoxide anions could be used ${ }^{10}$. A repertoire of chemiluminescent probes was also developed to detect specific ROS species with high sensitivity, such as luciferin-based probes ${ }^{15}, 16$. These have the advantage of being compatible with in vivo imaging but can't be used to map ROS production with specific cell types. Finally, this protocol can be applied to other types of organoids, for instance, colonic organoids derived from human biopsies. In this case, the culture growth medium should be adapted accordingly ${ }^{17}$. To further analyze the redox machinery within intestinal cells, the organoids culture and dissociation procedures described in this protocol can be combined with transcriptomic and proteomic approaches on whole organoids or Fluorescence activated cell sorted (FACS) organoids cells.

\section{ACKNOWLEDGMENTS:}

This work was supported by French National Research Agency (ANR) grant 17-CE14-0022 (iStress).

652 1. Aviello, G., Knaus, U. G. NADPH oxidases and ROS signaling in the gastrointestinal tract

DISCLOSURES:

The authors have nothing to disclose.

\section{REFERENCES:} review-article. Mucosal Immunology. 11 (4), 1011-1023 (2018).

2. Holmström, K. M., Finkel, T. Cellular mechanisms and physiological consequences of redoxdependent signalling. Nature Reviews Molecular Cell Biology. 15 (6), 411-421 (2014).

3. van der Post, S., Birchenough, G. M. H., Held, J. M. NOX1-dependent redox signaling potentiates colonic stem cell proliferation to adapt to the intestinal microbiota by linking EGFR and TLR activation. Cell Reports. 35 (1), 108949 (2021).

4. Schieber, M., Chandel, N. S. ROS function in redox signaling and oxidative stress. Current 
Biology. 24 (10), 453-462 (2014).

5. Myant, K. B. et al. ROS production and NF-KB activation triggered by RAC1 facilitate WNTdriven intestinal stem cell proliferation and colorectal cancer initiation. Cell Stem Cell. 12 (6), 761-773 (2013).

6. Juhasz, A. et al. NADPH oxidase 1 supports proliferation of colon cancer cells by modulating reactive oxygen species-dependent signal transduction. Journal of Biological Chemistry. 292 (19), 7866-7887 (2017).

7. Aviello, G., Knaus, U. G. ROS in gastrointestinal inflammation: Rescue Or Sabotage? British Journal of Pharmacology. 174 (12), 1704-1718 (2017).

8. Gomes, A., Fernandes, E., Lima, J. L. F. C. Fluorescence probes used for detection of reactive oxygen species. Journal of Biochemical and Biophysical Methods. 65 (2-3), 45-80 (2005).

9. Sato, T. et al. Single Lgr5 stem cells build crypt-villus structures in vitro without a mesenchymal niche. Nature. 459 (7244), 262-265 (2009).

10. Levy, A. et al. Innate immune receptor NOD2 mediates LGR5+ intestinal stem cell protection against ROS cytotoxicity via mitophagy stimulation. Proceedings of the National Academy of Sciences. 117 (4), 1994-2003 (2020).

11. Choi, H., Yang, Z., Weisshaar, J. C. Single-cell, real-time detection of oxidative stress induced in escherichia coli by the antimicrobial peptide CM15. Proceedings of the National Academy of Sciences of the United States of America. 112 (3), E303-E310 (2015).

12. Amri, F., Ghouili, I., Amri, M., Carrier, A., Masmoudi-Kouki, O. Neuroglobin protects astroglial cells from hydrogen peroxide-induced oxidative stress and apoptotic cell death. Journal of Neurochemistry. 140 (1), 151-169 (2017).

13. Ahn, H. Y. et al. Two-Photon Fluorescence Microscopy Imaging of Cellular Oxidative Stress Using Profluorescent Nitroxides. Journal of the American Chemical Society. 134 (10), 47214730 (2012).

14. Bidaux, G. et al. Epidermal TRPM8 channel isoform controls the balance between keratinocyte proliferation and differentiation in a cold-dependent manner. Proceedings of the National Academy of Sciences. 112 (26), E3345-E3354 (2015).

15. Van de Bittner, G. C., Dubikovskaya, E. A., Bertozzi, C. R., Chang, C. J. In vivo imaging of hydrogen peroxide production in a murine tumor model with a chemoselective bioluminescent reporter. Proceedings of the National Academy of Sciences. 107 (50), 21316 LP - 21321 (2010).

16. Rabbani, P. S., Abdou, S. A., Sultan, D. L., Kwong, J., Duckworth, A., Ceradini, D. J. In vivo imaging of reactive oxygen species in a murine wound model. Journal of Visualized Experiments. 141, e58450 (2018).

17. Sato, T. et al. Long-term expansion of epithelial organoids from human colon, adenoma, adenocarcinoma, and Barrett's epithelium. Gastroenterology. 141 (5), 1762-1772 (2011). 

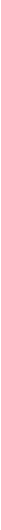

C
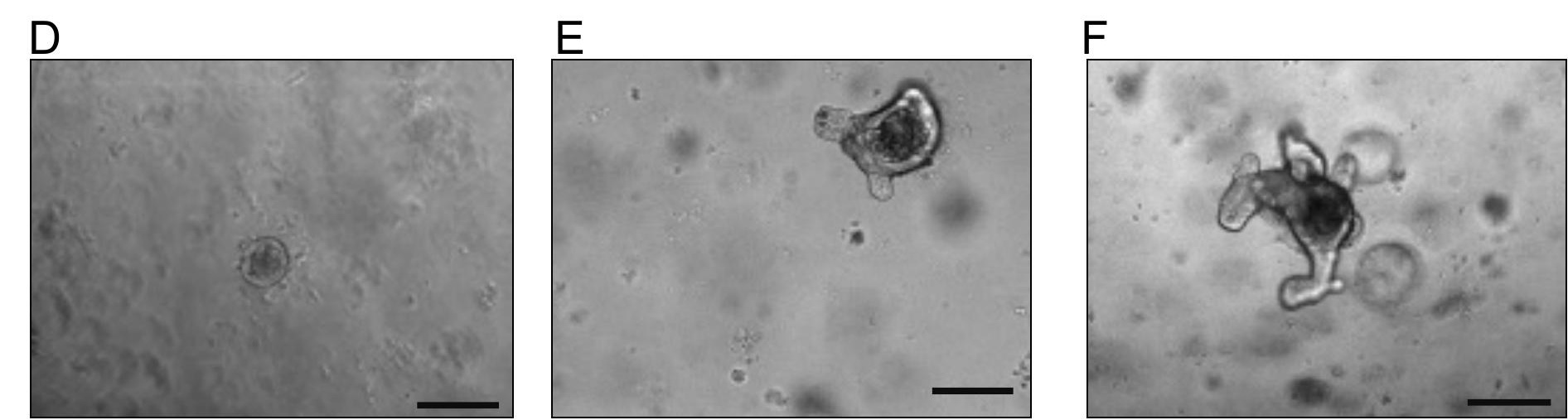

D 
A

\begin{tabular}{|l|l|l|}
\hline Control & Non-treated & NT \\
\hline Positive signal & tert-Butyl hydroperoxide & tBHP \\
\hline Middle signal & N-acetyl cysteine and tert-Butyl hydroperoxide & NAC-tBHP \\
\hline Negative signal & N-acetyl cysteine & NAC \\
\hline
\end{tabular}

B

NAC

tBHP

Fluorogenic

Probe

$+$

DAPI

Fluorogenic

Probe

DAPI

NS 

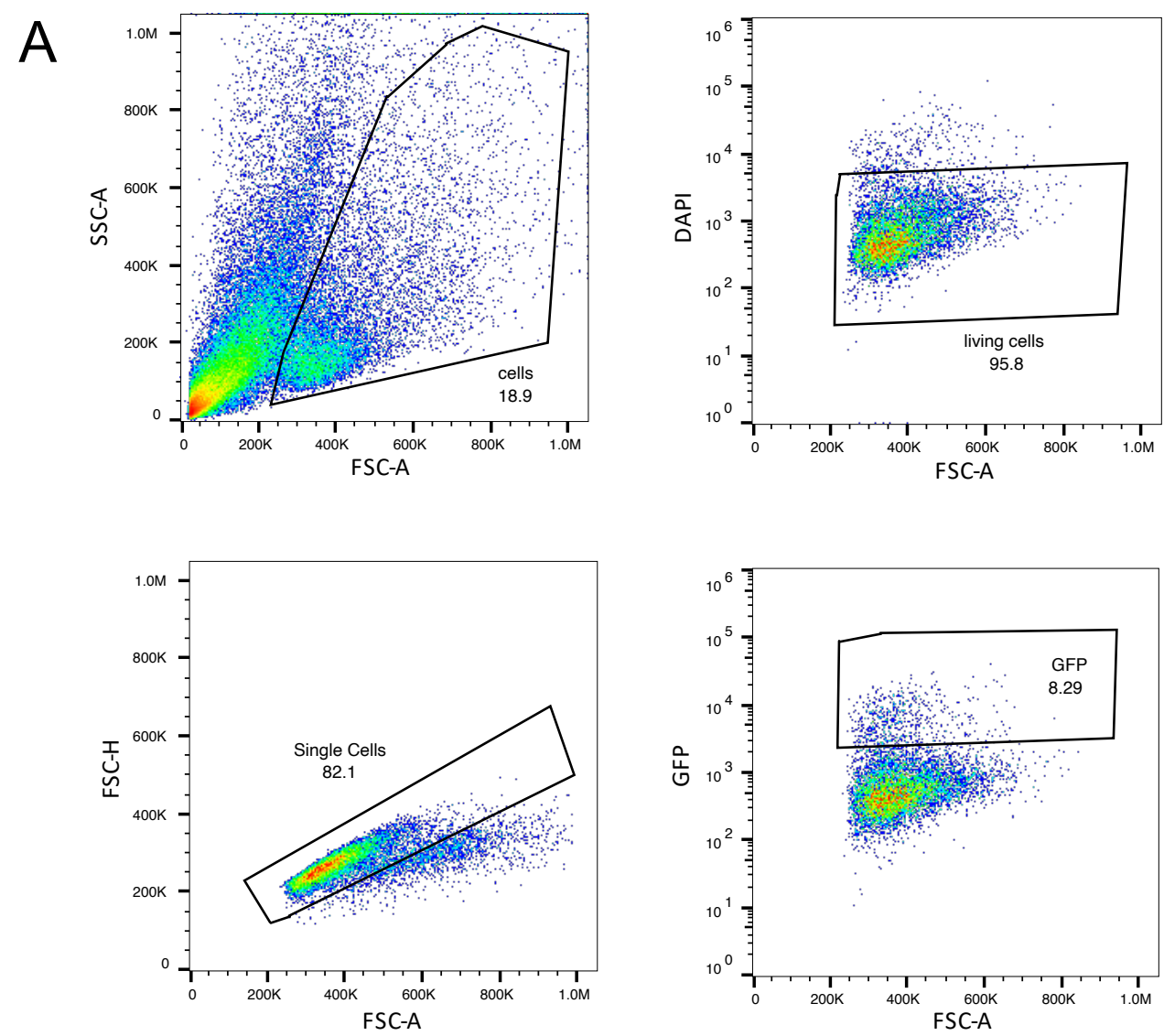

B

Total population
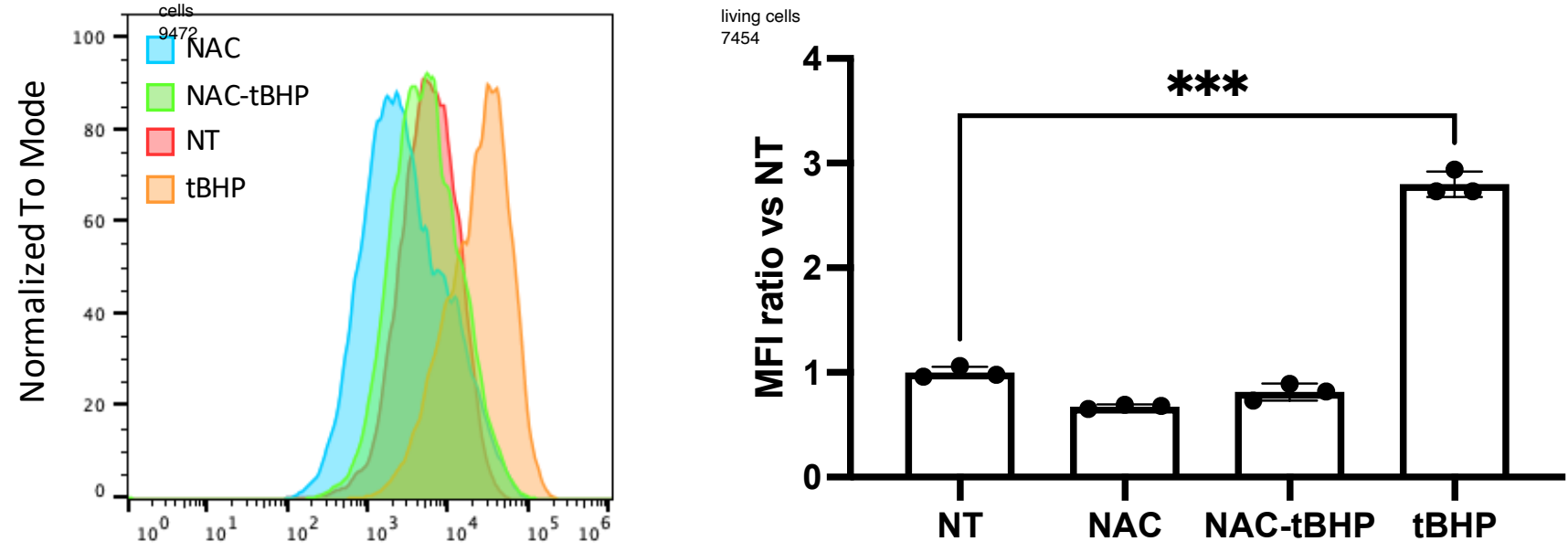

Fluorogenic Probe

C

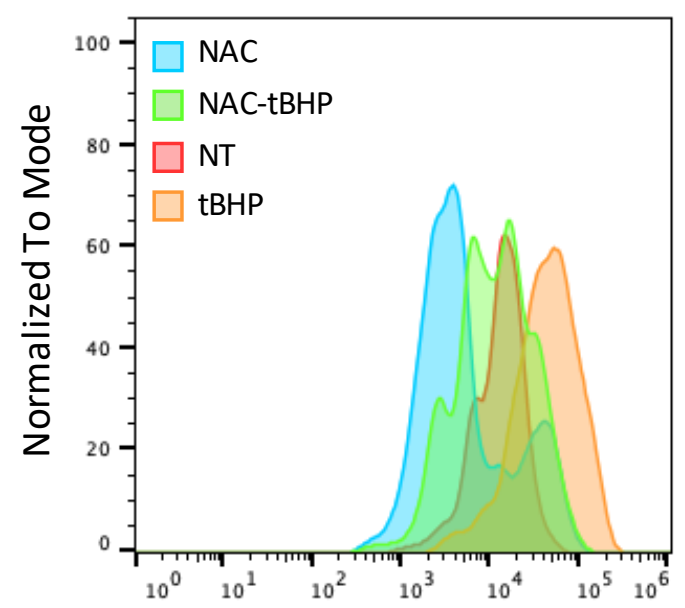

GFP+ cells

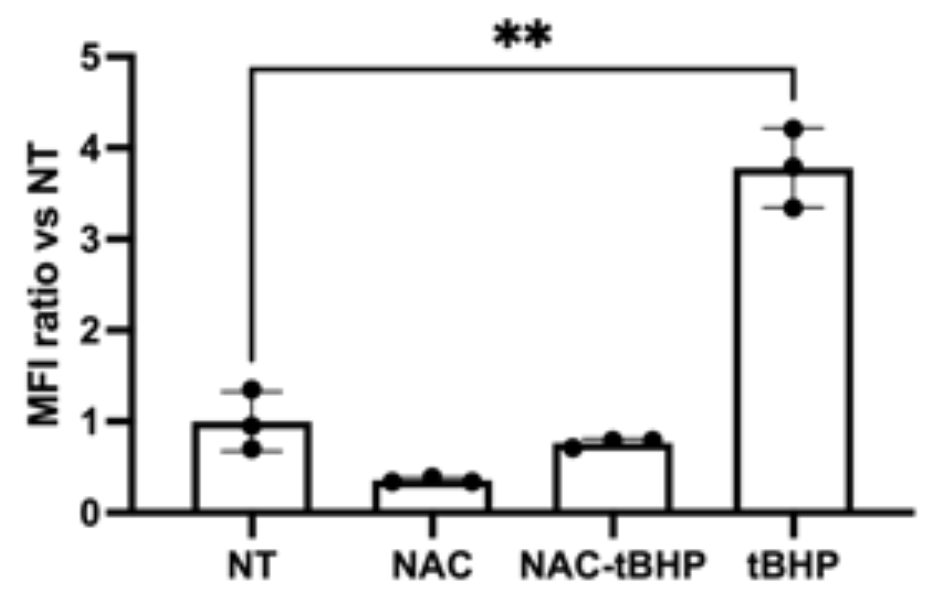

Fluorogenic Probe 


\section{Merge}

Nuclei

NT
Fluorogenic Probe

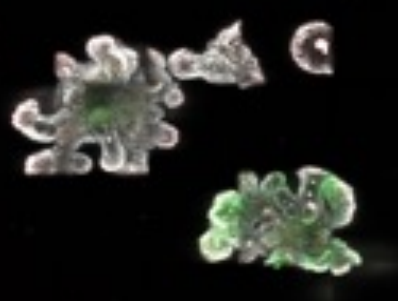

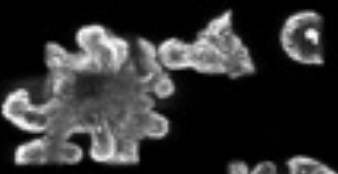

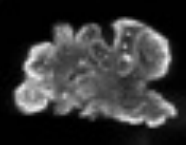

\section{NAC}
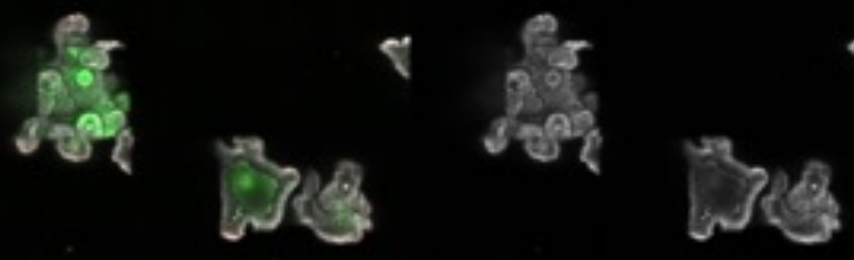

-

\section{tBHP}

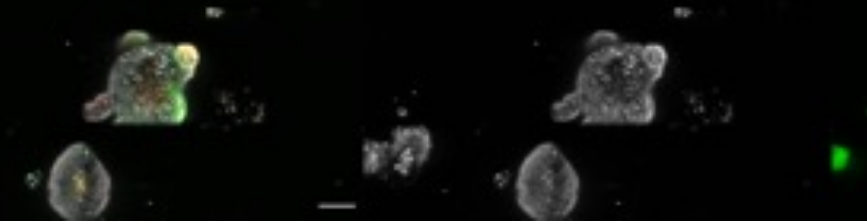

\section{NAC-tBHP}
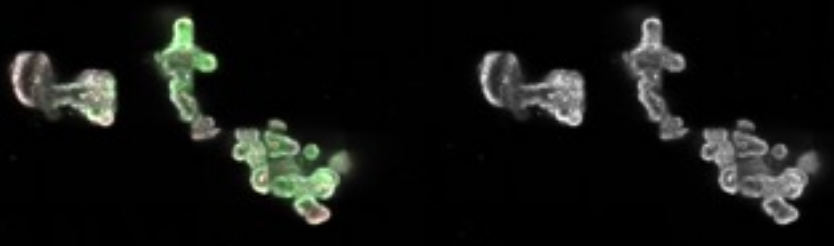
Mice

Company

Lgr5-EGFP-IRES-creERT2 (Lgr5-GFP) The Jackson Laboratory+A3:B3

\section{Growth culture medium}

Advanced DMEM F12 (DMEM/F12)

B-27 Supplement, minus vitamin A

GlutaMAX (glutamine)

Hepes

Murine EGF

murine Noggin

Murine R-spondin1

$\mathrm{N}-2$ Supplement

Penicillin-Streptomycin $(P / S)$

\section{Company}

ThermoFisher

ThermoFisher

ThermoFisher

ThermoFisher

$R \& D$

R\&D

R\&D

ThermoFisher

ThermoFisher

\begin{tabular}{|c|c|}
\hline Name of Material & Company \\
\hline $70 \mu \mathrm{m}$ cell strainer & Corning \\
\hline 96-well round bottom & Corning \\
\hline ball tip scissor & Fine Science Tools GMBH \\
\hline CellROX ${ }^{\circledR}$ Deep Red Reagent & ThermoFisher \\
\hline $\begin{array}{l}\text { DAPI (4',6-diamidino-2-phénylindole, } \\
\text { dichlorhydrate) (fluorgenic probe) }\end{array}$ & ThermoFisher \\
\hline $\begin{array}{l}\text { DPBS } 1 \times \text { no calcium no magnesium } \\
\text { (DPBS) }\end{array}$ & ThermoFisher \\
\hline $\begin{array}{l}\text { FLuoroBrite DMEM (DMEM no phenol } \\
\text { red) }\end{array}$ & ThermoFisher \\
\hline $\begin{array}{l}\text { Hoechst } 33342 \\
\text { Matrigel Growth Factor Reduced, } \\
\text { Phenol Red Free (Basement } \\
\text { Membrane Matrix) }\end{array}$ & $\begin{array}{l}\text { ThermoFisher } \\
\text { Corning }\end{array}$ \\
\hline$\mu$-Slide 8 Well chambers & Ibidi \\
\hline $\mathrm{N}$-acetylcysteine (NAC) & Sigma \\
\hline $\begin{array}{l}\text { tert-Butyl hydroperoxide } \\
\left.\text { (tBCHP)solution (70\%wt. In } \mathrm{H}_{2} \mathrm{O}_{2}\right)\end{array}$ & Sigma \\
\hline $\begin{array}{l}\text { TrypLE Express Enzyme (1X), no } \\
\text { phenol red (trypsin) }\end{array}$ & ThermoFisher \\
\hline UltraPure 0.5 M EDTA, pH8.0 & ThermoFisher \\
\hline Y-27632 & Sigma \\
\hline \multicolumn{2}{|l|}{ Programs and Equipment } \\
\hline $\begin{array}{l}\text { Attune NxT (Flow Cytometer) } \\
\text { Fiji/ImageJ }\end{array}$ & $\begin{array}{l}\text { ThermoFischer } \\
\text { https://imagej.net/software/fiii/dow } \\
\text { nloads }\end{array}$ \\
\hline FlowJo & $\overline{\mathrm{BD} \text { Bioscience }}$ \\
\hline Observer.Z1 & Zeiss \\
\hline Opterra (swept-field confocal) & Bruker \\
\hline $\begin{array}{l}\text { high speed EMCCD Camera Evolve } \\
\text { Delta } 512\end{array}$ & Photometrics \\
\hline Prism & GraphPad Software \\
\hline
\end{tabular}

Catalog Number

12634010

35050038

15630056

2028-EG-200

1967-NG/CF

3474-RS-050

17502048

15140122
Catalog Number

12587010

\section{Comments/Description}

\section{Stock Concentration}

stock: $50 \mathrm{X}$

stock: $100 \mathrm{x}$

stock: $1 \mathrm{M}$

stock: $500 \mu \mathrm{g} / \mathrm{mL}$ in PBS

stock: $100 \mu \mathrm{g} / \mathrm{mL}$ in PBS

stock: $50 \mu \mathrm{g} / \mathrm{mL}$ in PBS

stock: $100 \mathrm{x}$

stock: $100 x(10,000$ units $/ \mathrm{mL}$ of penicillin and $10,000 \mu \mathrm{g} / \mathrm{mL}$ of streptomycin)

\author{
Catalog Number Comments/Description \\ 352350 \\ 3799 \\ 14086-09 \\ C10422 \\ D1306 \\ stock at $10 \mathrm{mg} / \mathrm{mL}$
}

14190144

A1896701

H3570

356231

80826

A9165

458139

12604013

15575020

Y0503

Rock-inhibitor to be used to minimize cell death upon tissue dissociation

\section{Comments/Description}

Flow cytometer analyzer images generation

FACS analysis

confocal system

statistical analysis 